\title{
BRCA1 shields vascular smooth muscle cells from oxidative stress
}

\author{
Fina Lovren, PhD, ${ }^{\mathrm{a}, \mathrm{e}}$ Yi Pan, MD, ${ }^{\mathrm{a}, \mathrm{e}}$ Adrian Quan, MPhil, ${ }^{\mathrm{a}, \mathrm{e}}$ Krishna K. Singh, PhD, ${ }^{\mathrm{a}, \mathrm{e}}$ Rishad Khan, ${ }^{\mathrm{a}, \mathrm{e}}$ \\ Nandini Gupta, MD, ${ }^{\text {h }}$ Christine Brezden-Masley, MD, PhD, ${ }^{\text {be, }, \mathrm{f}}$ Hwee Teoh, $\mathrm{PhD},{ }^{\mathrm{a}, \mathrm{c}, \mathrm{e}}$ \\ Mark D. Wheatcroft, BSc, MBChB, MD, ${ }^{\text {d,e,g }}$ Mohammed Al-Omran, MD, MSc, ${ }^{\text {de, }, \mathrm{g}}$ and \\ Subodh Verma, MD, $\mathrm{PhD}^{\mathrm{a}, \mathrm{e}, \mathrm{g}}$
}

\begin{abstract}
Background: Excessive production of reactive oxygen species (ROS), in part via upregulation of DNA damage pathways, is a central mechanism governing pathologic activation of vascular smooth muscle cells (VSMCs). We hypothesized that the breast cancer 1, early onset (BRCA1) gene that is involved in cellular resistance to DNA damage limits ROS production and oxidative stress in VSMCs.
\end{abstract}

\begin{abstract}
Methods: We evaluated basal and $\mathrm{H}_{2} \mathrm{O}_{2}$-stimulated expression of BRCA1 in human aortic smooth muscle cells (HASMCs). In vitro gain-of-function experiments were performed in BRCA1 adenovirus (Ad-BRCA1)-transfected HASMCs. ROS production and expression of Nox1 and its key regulatory subunit p47phox, key components of the ROS-generating nicotinamide adenine dinucleotide phosphate $(\mathrm{NADPH})$ oxidase system, were evaluated. In vivo gain-of-function experiments were performed in spontaneously hypertensive $(\mathrm{SHR})$ rats treated with Ad-BRCA1 $\left(5 \times 10^{10} \mathrm{IU} / \mathrm{rat}\right)$. Blood pressure, vascular ROS generation, Nox1, and p47phox expression were measured.
\end{abstract}

Results: BRCA1 was constitutively expressed in murine, rat, and human smooth muscle cells (SMCs). $\mathrm{H}_{2} \mathrm{O}_{2}$ significantly reduced BRCA1 expression with a resultant increase in ROS generation. BRCA1-overexpressing HASMCs were protected against $\mathrm{H}_{2} \mathrm{O}_{2}$-induced ROS generation, in part, via downregulation of the ROS-producing NADPH oxidase subunits Nox 1 and p47phox. Ad-BRCA1 treatment in SHR rats was associated with a sustained increase in aortic BRCA1 expression, lower aortic ROS production, reduced $\gamma \mathrm{H} 2 \mathrm{~A} . \mathrm{X}$ levels, greater RAD51 foci, and decreases in blood pressure.

Conclusions: BRCA1 is a novel and previously unrecognized target that may shield VSMCs from oxidative stress by inhibiting NADPH Nox1-dependent ROS production. Gene- and/or cell-based approaches that improve BRCA1 bioavailability may represent a new approach in the treatment of diverse vascular diseases associated with an aberrant VSMC phenotype. (J Thorac Cardiovasc Surg 2014;147:1946-55)

Supplemental material is available online.

\footnotetext{
From the Divisions of Cardiac Surgery, ${ }^{\mathrm{a}}$ Medicine \& Hematology-Oncology, ${ }^{\mathrm{b}}$ Endocrinology \& Metabolism, ${ }^{\mathrm{c}}$ and Vascular \& Endovascular Surgery, ${ }^{\mathrm{d}}$ Keenan Research Centre in the Li Ka Shing Knowledge Institute, ${ }^{\mathrm{e}}$ St. Michael's Hospital, Toronto, Ontario, Canada; Departments of Medicine ${ }^{\mathrm{f}}$ and Surgery, ${ }^{\mathrm{g}}$ University of Toronto, Toronto, Ontario, Canada; and University of Ottawa Heart Institute, Ottawa, Ontario, Canada.

This work was supported by grants from the Heart and Stroke Foundation of Canad and the Canadian Institutes of Health Research (to S.V.), an unrestricted Cardiology-Oncology Education Program grant from Sanofi-Aventis Canada Inc (to C.B-M. and S.V.) and unrestricted grants from Roche Canada (to C.B-M. and S.V.), Abbott Canada (to S.V.) and AstraZeneca Canada (to S.V.). S.V. is the Canada Research Chair in Atherosclerosis at the University of Toronto.

Disclosures: Authors have nothing to disclose with regard to commercial support.

Received for publication July 17, 2013; revisions received Sept 23, 2013; accepted for publication Sept 30, 2013; available ahead of print Nov 18, 2013.

Address for reprints: Subodh Verma, MD, PhD, Division of Cardiac Surgery, St. Michael's Hospital, Suite 8-003, Bond Wing, 30 Bond St, Toronto, Ontario, Canada, M5B 1W8 (E-mail: vermasu@smh.ca).

$0022-5223 / \$ 36.00$

Copyright $(\odot) 2014$ by The American Association for Thoracic Surgery

http://dx.doi.org/10.1016/j.jtcvs.2013.09.060
}

VSMCs are responsible for arterial contractile tone and regulating blood pressure and flow to meet metabolic demands. ${ }^{1-4}$ In response to a variety of acquired risk factors, and/or alterations in shear stress, VSMCs undergo pathologic activation, with marked changes in structure and function, which have been causally related to several cardiovascular diseases, including hypertension, atherosclerosis, neointimal hyperplasia/restenosis, and aneurysm formation. ${ }^{1-7}$

One of the best described mechanisms of VSMC activation is oxidative stress, due mainly to increased production of reactive oxygen species (ROS) such as superoxide, and/ or a reduced ability to quench ROS due to a diminished bioavailability of nitric oxide (NO) or antioxidant enzymes. ${ }^{1,2}$ ROS stimulate various signaling pathways within the VSMC, including mitogen-activated protein kinases (MAPK), tyrosine kinases, Rho kinase, and transcription factors such as nuclear factor kappa B, activator protein-1, and hypoxia-inducible factor 1 , in addition to stimulating protooncogene and proinflammatory gene expression. With progressive exposure to ROS and oxidative stress, cells undergo irreversible DNA damage resulting 


\section{Abbreviations and Acronyms \\ DHE = dihydroethidium \\ DSB $=$ double-strand breaks \\ GAPDH $=$ glyceraldehyde-3-phosphate dehydrogenase}

HASMC = human aortic smooth muscle cells

MAPK $=$ mitogen-activated protein kinases

MASMC $=$ mouse aortic smooth muscle cells

MOI = multiplicity of infection

$\mathrm{NADPH}=$ nicotinamide adenine dinucleotide phosphate

PCR = polymerase chain reaction

RASMC $=$ rat aortic smooth muscle cells

ROS = reactive oxygen species

SHR = spontaneously hypertensive rat

SMC = smooth muscle cells

VSMC = vascular smooth muscle cells

WKY $=$ Wistar-Kyoto rat

in apoptosis and/or necrosis. ${ }^{1,2}$ Therefore, identifying the mechanisms of the deleterious effects of oxidative stress can serve as a novel approach to limit VSMC activation, and the associated pathologic consequences.

The breast cancer 1, early onset (BRCA1) gene functions as a caretaker of genome stability and exerts multiple effects on key cellular processes involved in DNA damage repair, transcriptional regulation, ubiquitination, and cell cycle control. ${ }^{8,9}$ Although BRCA1 is widely known as a tumor suppressor gene in breast and ovarian cancer syndromes, accumulating evidence suggests that BRCA1 and BRCA2 may function to limit oxidative stressinduced DNA damage in other tissues, and through this mechanism may afford protection against ischemic and genotoxic cardiac failure, atherosclerosis, and sepsis. ${ }^{10-14}$

Because oxidative stress is an important trigger for DNA damage, and BRCA1 is well described to limit oxidative and genotoxic injury, we hypothesized that it may function in a similar fashion within VSMCs, guarding them against oxidative injury and ROS production. This study shows that BRCA1 may serve to limit VMSC ROS production in part through an nicotinamide adenine dinucleotide phosphate (NADPH)-dependent pathway, and that in vivo BRCA1 gene delivery reduces aortic oxidative stress and blood pressure in hypertensive animals.

\section{METHODS}

\section{Reagents}

Human aortic smooth muscle cells (HASMCs) from healthy donors were purchased from Lonza (Walkmersville, Md) and cultured in medium from Gibco (Carlsbad, Calif). Antibodies directed against glyceraldehyde3-phosphate dehydrogenase (GAPDH), NADPH oxidase catalytic subunit 1 (Nox1), and p47phox were from Millipore (Etobicoke, Ontario, Canada), Cell Signaling Technology, Inc (Danvers, Mass), and Santa Cruz
Biotechnology (Santa Cruz, Calif), respectively. The custom-designed BRCA1 adenovirus (Ad-BRCA1; containing the complete coding sequence of human BRCA1 [accession no. NM_007294.2]) and the control adenovirus Ad-null were from Vector BioLabs (Philadelphia, Penn). Both were under the control of the cytomegalovirus promoter. Reagents for standard and real-time polymerase chain reaction (qPCR) were from Applied Biosystems (Burlington, Ontario, Canada) and Qiagen (Toronto, Ontario, Canada). Immunoblotting reagents and 6-chloromethyl$2^{\prime}, 7^{\prime}$-dichlorodihydrofluorescein diacetate, acetyl ester (CM-DCF) were from Life Technologies (Burlington, Ontario, Canada). The remaining reagents were from Sigma (Oakville, Ontario, Canada).

\section{Animals}

Male spontaneously hypertensive (SHR) and normotensive WistarKyoto (WKY) rats (6-8 weeks old) were from Charles River Laboratories International, Inc (Wilmington, Mass). When necessary, rats were injected with Ad-null or Ad-BRCA1 $\left(5 \times 10^{10} \mathrm{IU} / \mathrm{rat}\right)$ via their tail veins. Male ( $\sim 8$ weeks old) C57B1/6J mice were from the Jackson Laboratory (Bar Harbor, Me). All animal procedures were in accordance with the guidelines of the Canadian Council on Animal Care and were approved by the St. Michael's Hospital Animal Care Committee.

\section{Radiotelemetry Recordings}

Radiotelemetric catheters (model PA-C10; Data Sciences International, St. Paul, Minn) were implanted in the abdominal aorta of 6-week-old rats at the Charles River Laboratories (Raleigh, NC). Rats were exported to St. Michael's Hospital a week later where they were allowed to acclimatize for 7 days before they were administered either Ad-null or Ad-BRCA1 $\left(5 \times 10^{10} \mathrm{IU} / \mathrm{rat}\right)$ via the tail vein. Arterial pressure and heart rate data were acquired for 1 minute every 6 hours by the Dataquest A.R.T. 4.10 software via RPC-1 receivers (Data Sciences International).

\section{Isolation and Culture of Rat and Mouse Aortic Smooth Muscle Cells}

Rat and mouse aortic smooth muscle cells (RASMCs and MASMCs, respectively) were isolated by enzymatic dispersion of whole aortas.

\section{SMC RNA}

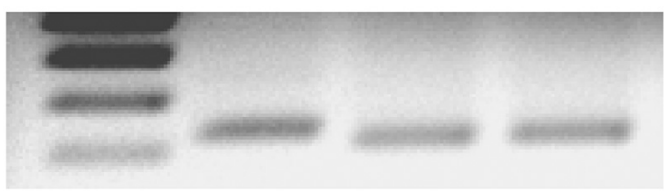

BRCA1 (114 bp)

\section{A MW Mouse Rat Human}

SMC Protein

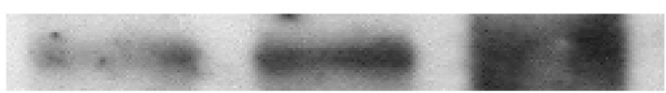

BRCA1 (240 kD)

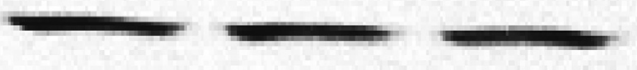

GAPDH (38 kD)

\section{B Mouse Rat Human}

FIGURE 1. BRCA1 is constitutively expressed in aortic SMCs. A, Representative agarose gel image and (B) immunoblot confirming the constitutive presence of BRCA1 transcript and protein in C57B1/6J MASMCs, WKY RASMCs and HASMCs. $\mathrm{n}=5$ per group. $S M C$, Smooth muscle cells; $B R C A 1$, breast cancer 1 , early onset gene; $M W$, molecular weight marker $G A P D H$, glyceraldehyde-3-phosphate dehydrogenase. 

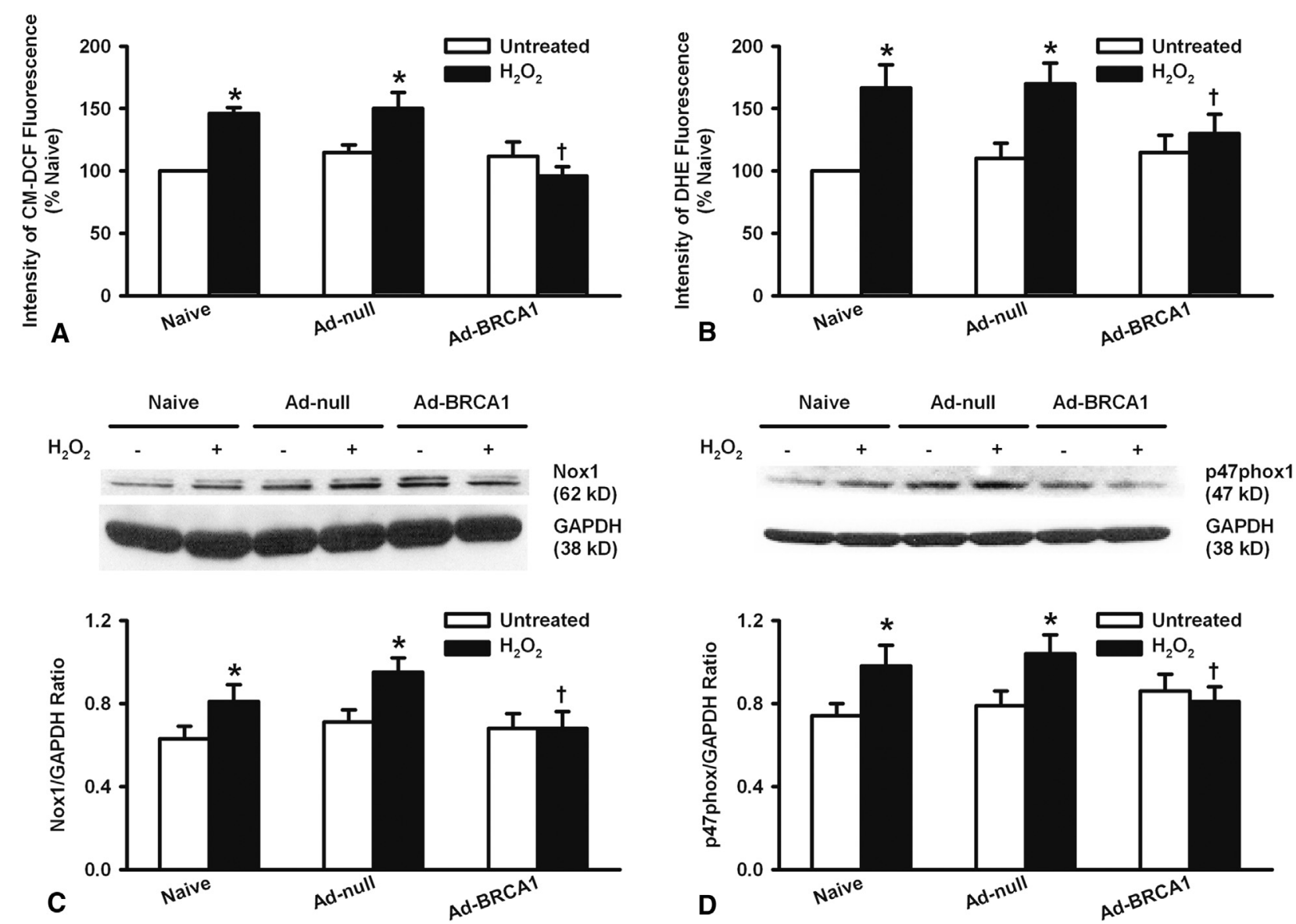

FIGURE 2. BRCA1 overexpression reduces oxidative stress in HASMCs via the NADPH oxidase system. ROS production was determined by (A) CM-DCF and (B) DHE fluorescence in HASMCs stressed for 24 hours with $\mathrm{H}_{2} \mathrm{O}_{2}$. Representative Western blots and semiquantitative data for the NADPH oxidase subunits (C) Nox1 and (D). p47phox in naive and adenovirus-treated HASMCs. GAPDH acted as the housekeeping protein. $\mathrm{n}=5$ per group. ${ }^{*} P<.05$ versus the corresponding untreated group; $\dagger P<.05$ versus the $\mathrm{H}_{2} \mathrm{O}_{2}$-treated Ad-null-transfected group. $C M$ - $D C F$, 6-Chloromethyl2',7'-dichlorodihydrofluorescein diacetate, acetyl ester; Ad-null, control adenovirus; Ad-BRCA1, BRCA1 adenovirus; DHE, dihydroethidium; $G A P D H$, glyceraldehyde-3-phosphate dehydrogenase.

Briefly, aortas were opened longitudinally, the endothelial layer gently scraped, and the media removed from the underlying adventitia into a cocktail of collagenase $(1 \mathrm{mg} / \mathrm{mL})$, soybean trypsin inhibitor $(1 \mathrm{mg} / \mathrm{mL})$, and elastase $(0.744 \mathrm{U} / \mathrm{mL})$ in Hanks balanced salt solution for 60 minutes at $37^{\circ} \mathrm{C}$. RASMCs so isolated were cultured in Dulbecco's modified Eagle medium/Nutrient Mixture F-12 medium supplemented with $20 \%$ fetal bovine serum and antibiotics. Studies were performed on SMCs from passages 4 to 7 . Subconfluent cell cultures were rendered quiescent by serum deprivation 24 hours before initiation of all experiments.

\section{ROS Production}

Generation of ROS was assessed in naive or adenovirus-transfected HASMCs and RASMCs via CM-DCF and dihydroethidium (DHE) fluorescence. ROS signals were captured by confocal microscopy after SMCs had been stimulated with $\mathrm{H}_{2} \mathrm{O}_{2}(100 \mu \mathrm{M})$ for 24 hours and incubated in the dark for either 45 minutes with $10 \mu \mathrm{M} \mathrm{CM}$-DCF or 30 minutes with $5 \mu \mathrm{M}$ DHE.

Aortic ROS production was assessed by lucigenin-enhanced chemiluminescence. Briefly, freshly isolated aortic segments were incubated for 30 minutes at $37^{\circ} \mathrm{C}$ in $95 \% \mathrm{O}_{2} / 5 \% \mathrm{CO}_{2}$-aerated modified Krebs-HEPES buffer containing in mmol/L: $\mathrm{NaCl} 99.01, \mathrm{KCl} 4.69, \mathrm{CaCl}_{2} 1.87, \mathrm{MgSO}_{4}$ 1.20, Na-HEPES 20.0, $\mathrm{K}_{2} \mathrm{HPO}_{4}$ 1.03, $\mathrm{NaHCO}_{3}$ 25.0, and D-(+)-glucose
11.1 ( $\mathrm{pH}$ 7.4). Aortic segments were subsequently transferred to 24 -well plates; each well contained $2 \mathrm{~mL}$ of Krebs-HEPES buffer with $5 \mu \mathrm{mol} / \mathrm{L}$ lucigenin. Luminescence for each aortic segment was recorded and normalized to its dry weight.

\section{NADPH Oxidase Activity}

RASMCs were washed in ice-cold phosphate buffered saline and resuspended in a lysis buffer $\left(\mathrm{pH}\right.$ 7.4) containing $\mathrm{KH}_{2} \mathrm{PO}_{4}(20 \mathrm{mmol} / \mathrm{L})$, ethylene glycol tetraacetic acid (EGTA; $1 \mathrm{mmol} / \mathrm{L}$ ), and protease inhibitors. NADPH $(0.1 \mathrm{mmol} / \mathrm{L})$ was added to $200 \mu \mathrm{L}$ of suspensions containing $50 \mu \mathrm{L}$ of RASMC or thoracic aorta homogenate, $5 \mathrm{mmol} / \mathrm{L}$ lucigenin, and assay buffer (in mmol/L: $\mathrm{KH}_{2} \mathrm{PO}_{4} 50$, EGTA 1 , sucrose $150 ; \mathrm{pH} 7.4$ ) before chemiluminescence was recorded.

\section{RNA Analyses}

RNA extracted from whole tissues and intact SMCs were reverse transcribed with a TaqMan reverse transcription kit before qPCR was performed on the StepOnePlus Real-Time PCR System using the TaqMan Universal PCR Mix. Gene expression data were analyzed by the $\Delta \Delta \mathrm{Ct}$ method. In some cases, RNA from intact SMCs and liver samples were examined via traditional PCR methods. 

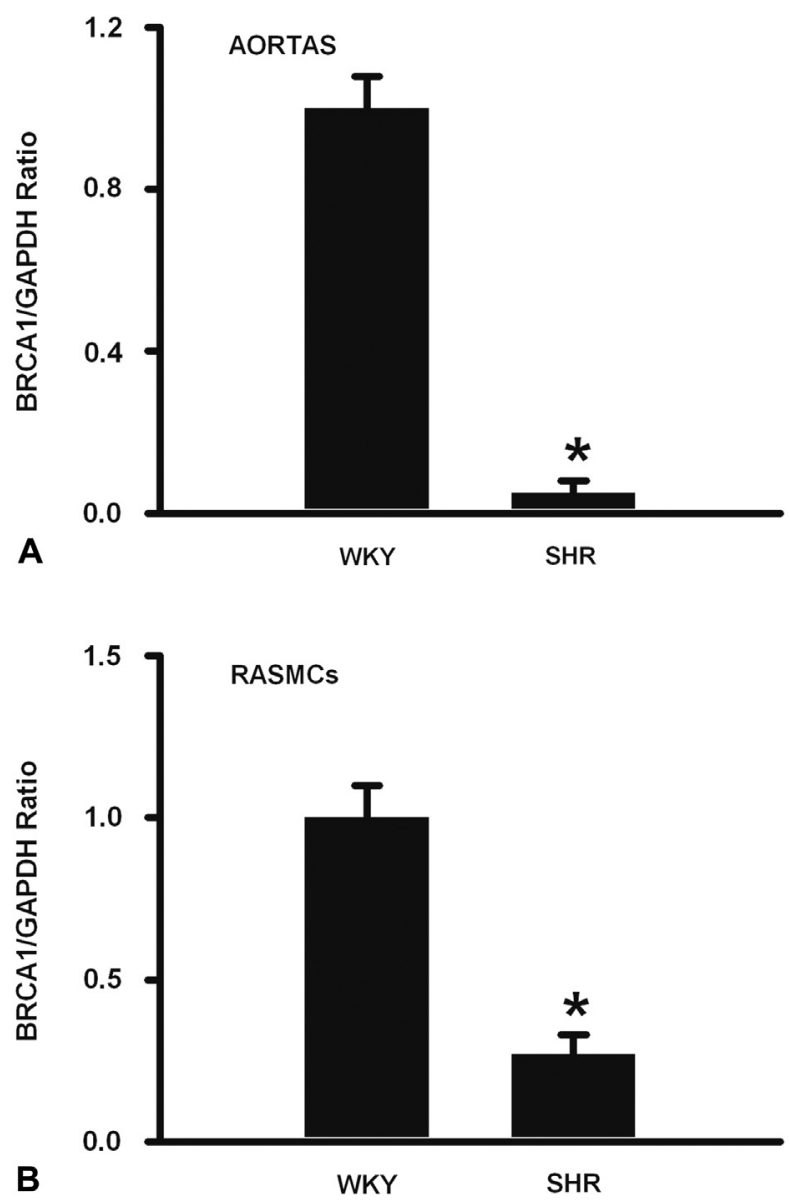

FIGURE 3. Aortas and RASMCs from SHR rats express less BRCA1 than those from WKY rats. BRCA1 transcripts in (A) WKY and SHR rat aorta and (B) RASMC homogenates were assessed via qPCR. $\mathrm{n}=5$ per group. $* P<.05$ versus WKY group. BRCA1, Breast cancer 1, early onset gene; $G A P D H$, glyceraldehyde-3-phosphate dehydrogenase; WKY, WistarKyoto rat; $S H R$, spontaneously hypertensive rat; RASMC, rat aortic smooth muscle cells.

\section{Immunoblotting}

Protein lysates were separated on $4 \%$ to $20 \%$ polyacrylamide gels and transferred to nitrocellulose membranes, which were subsequently probed with antibodies directed against Nox1, p47phox, and GAPDH and incubated with the appropriate horseradish peroxidase-tagged secondary antibody. Immunoblotting signals were revealed with chemiluminescence and quantified by densitometry.

\section{Transfection}

HASMCs and RASMCs were incubated for 24 hours with 10 multiplicity of infection (MOI) of Ad-BRCA1 or Ad-null. Transfection efficiency was evaluated by qPCR.

\section{Histology}

Whole aortas were snap frozen in optimal cutting temperature compound and sectioned $(5 \mu \mathrm{m})$ before being processed for routine immunohistochemical examination. Sections were incubated with antibodies directed against either $\gamma \mathrm{H} 2 \mathrm{~A}$.X (1:50; Thermo Scientific, Waltham, Mass) or RAD51 (1:50; Millipore). Nuclei positive for $\gamma \mathrm{H} 2 \mathrm{~A} . \mathrm{X}$ or RAD51 were quantified in 5 random fields.

\section{Statistics}

Data are presented as means (standard error of the mean) and were analyzed with either the Student $t$ test or 1-way analysis of variance followed by the Bonferroni multiple comparisons procedure for all secondary $t$ tests of means.

\section{RESULTS}

\section{BRCA1 Is Constitutively Expressed in SMCs}

We confirmed that BRCA1 is constitutively expressed at both the transcript and protein levels in aortic SMCs from mice, rats, and humans (Figure 1).

\section{BRCA1 Guards SMCs Against Oxidative Stress}

Increased oxidative stress is an essential mechanism leading to SMC dysfunction in various cardiovascular disease states. To evaluate the relationship between BRCA1 and oxidative stress, HASMCs were treated with the prototypical oxidant $\mathrm{H}_{2} \mathrm{O}_{2}$ and changes in BRCA1 expression were measured. BRCA1 expression was markedly attenuated by $\mathrm{H}_{2} \mathrm{O}_{2}$ in a concentration-dependent manner with an approximately $40 \%$ reduction in BRCA1 expression noted at $100 \mu \mathrm{M} \mathrm{H}_{2} \mathrm{O}_{2}$ (Figure E1). Accordingly, this concentration of $\mathrm{H}_{2} \mathrm{O}_{2}$ was used in the subsequent experiments.

To elucidate whether BRCA1 protects SMCs from oxidative stress, ROS production was measured in BRCA1-overexpressing HASMCs exposed to $\mathrm{H}_{2} \mathrm{O}_{2}$ via CM-DCF fluorescence and DHE staining (Figure 2, $A$ and $B$ ). The elaborated ROS production elicited by $\mathrm{H}_{2} \mathrm{O}_{2}$ was completely inhibited in Ad-BRCA1-transfected HASMCs (Figure 2, A). Superoxide generation, as assessed by DHE staining, was also markedly lower in $\mathrm{H}_{2} \mathrm{O}_{2}$-stressed BRCA1 overexpressing HASMCs relative to the corresponding Ad-null-treated group (Figure 2, B).

ROS generation in SMCs is largely controlled by the NADPH oxidase (Nox) system. Nox1 is found abundantly in SMCs and requires the regulatory subunit $\mathrm{p} 47 \mathrm{phox}$ for its activity. We observed that, whereas $\mathrm{H}_{2} \mathrm{O}_{2}$ caused a significant increase in Nox 1 and p47phox expression in Ad-null-treated HASMCs, this effect was attenuated in Ad-BRCA1-transfected HASMCs (Figure 2, $C$ and $D$ ). These data suggest that in SMCs, BRCA1 serves to attenuate $\mathrm{H}_{2} \mathrm{O}_{2}$-induced ROS production, in part, through a Nox1-dependent pathway.

\section{SMCs From Hypertensive Animals Exhibit Reduced BRCA1 Expression and Increased Susceptibility to Oxidative Stress}

Increased VSMC oxidative stress has been implicated as a causal mechanism of hypertension in various animal models, including the SHR rat. To extend our in vitro observations, we tested BRCA1 expression and ROS production in aortic SMCs isolated from SHR and WKY control rats. We also performed gain-of-function in vivo studies to determine if 


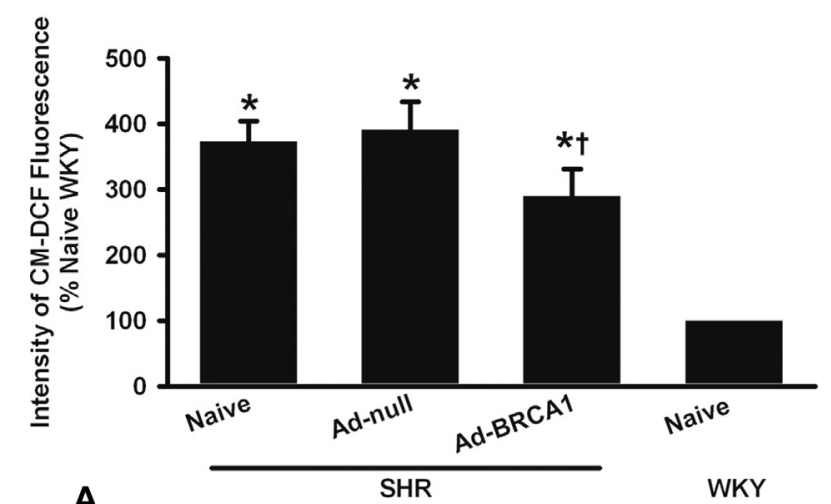

A
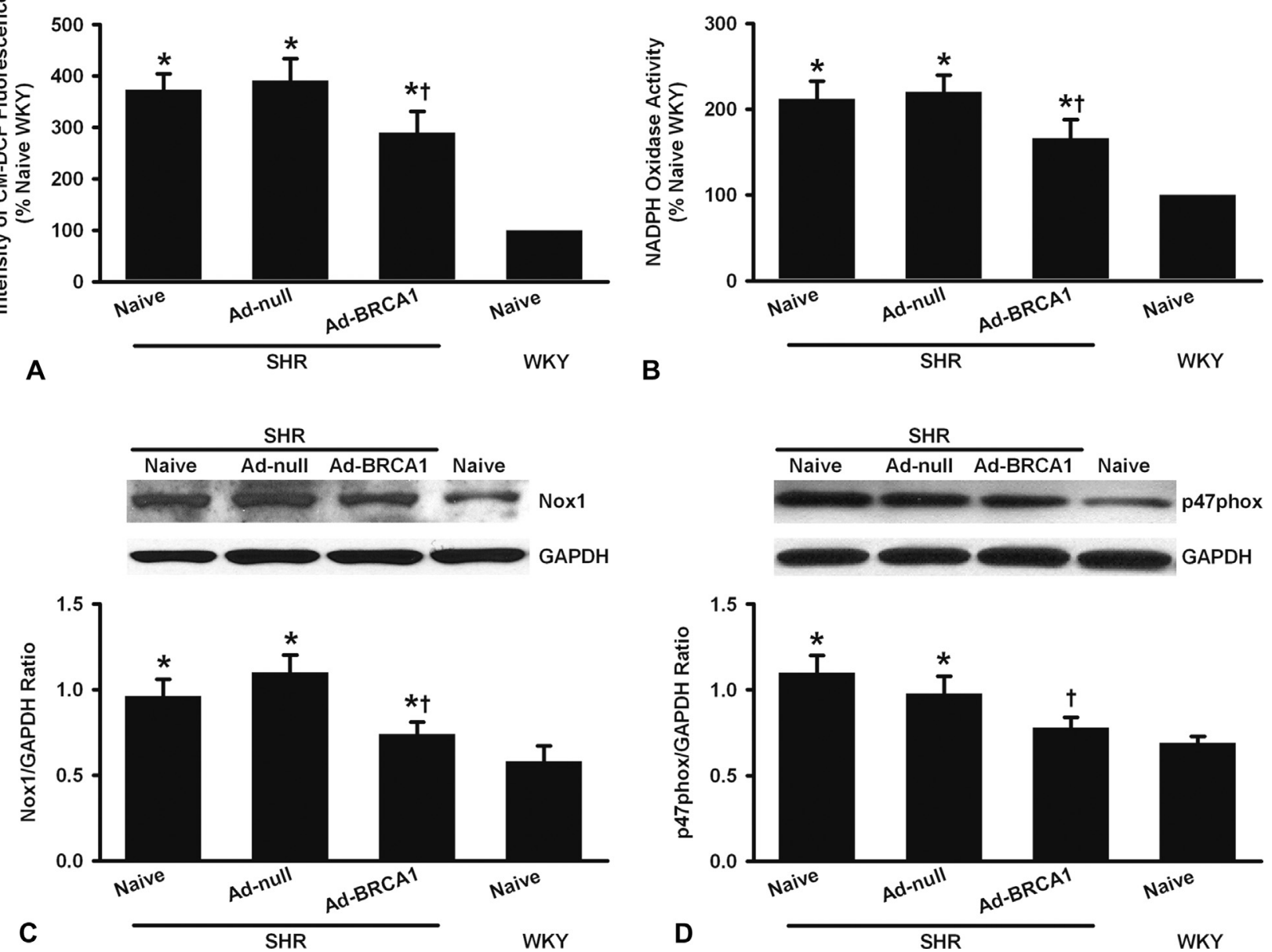

FIGURE 4. BRCA1 overexpression reduces oxidative stress in SHR RASMCs via the NADPH oxidase system. A, ROS production and (B) NADPH oxidase activity were assessed by CM-DCF fluorescence and lucigenin-enhanced chemiluminescence, respectively, in homogenates of WKY RASMCs and adenovirustransfected SHR RASMCs ( $\mathrm{n}=6$ per group). Representative Western blots and semiquantitative data for the NADPH oxidase subunits (C) Nox1 and (D) $\mathrm{p} 47 \mathrm{phox}$ in WKY and SHR RASMC homogenates. $\mathrm{n}=5$ per group. ${ }^{*} P<.05$ versus WKY group; $\dagger P<.05$ versus Ad-null SHR group. $C M-D C F, 6$-Chloromethyl-2' $7^{\prime}$ dichlorodihydrofluorescein diacetate, acetyl ester; WKY, Wistar-Kyoto rat; Ad-null, control adenovirus; Ad-BRCA1, BRCA1 adenovirus; SHR, spontaneously hypertensive rat; $G A P D H$, glyceraldehyde-3-phosphate dehydrogenase; $N A D P H$, nicotinamide adenine dinucleotide phosphate.

systemic overexpression of BRCA1 would reduce SMC oxidative stress and lower blood pressure in SHR rats.

Basal BRCA1 transcript levels, as measured by qPCR, were significantly lower in aortas and RASMCs from SHR rats compared with WKY rats (Figure 3), supporting the notion that BRCA1 may play a fundamental role in the spontaneous development of hypertension in SHR rats.

An 18-fold difference in BRCA1 expression was observed between Ad-BRCA1-transfected SHR RASMCs and Ad-null-transfected SHR RASMCs, thus confirming successful transfection (Figure E2). CM-DCF fluorescence measurements indicated that the ROS levels in naive RASMCs from SHR rats were markedly higher than those in naive RASMCs from WKY rats (Figure 4, $A$ ). ROS levels in BRCA1-overexpressing SHR RASMCs were appreciably lower than those in naive and Ad-null-transfected SHR RASMCs (Figure 4, $A$ ).
We then investigated the role of NADPH oxidase in modulating the protective effects of BRCA1 to limit ROS production in RASMCs from SHR rats. NADPH oxidase activity was significantly higher in naive RASMCs from SHR rats compared with WKY rats and, importantly, BRCA1 overexpression attenuated the enhanced NADPH activity noted in SHR RASMCs (Figure 4, B). Correspondingly, Nox1 and p47phox protein levels, highest in naive and Ad-null-transfected SHR RASMCs, were markedly lower in BRCA1-overexpressing SHR RASMCs (Figure 4, $C$ and $D$ ).

\section{In Vivo BRCA1 Gene Delivery Attenuates Vascular Oxidative Stress and Lowers Blood Pressure in Hypertensive Animals}

Because vascular oxidative stress plays an important role in the development of hypertension, we hypothesized that 


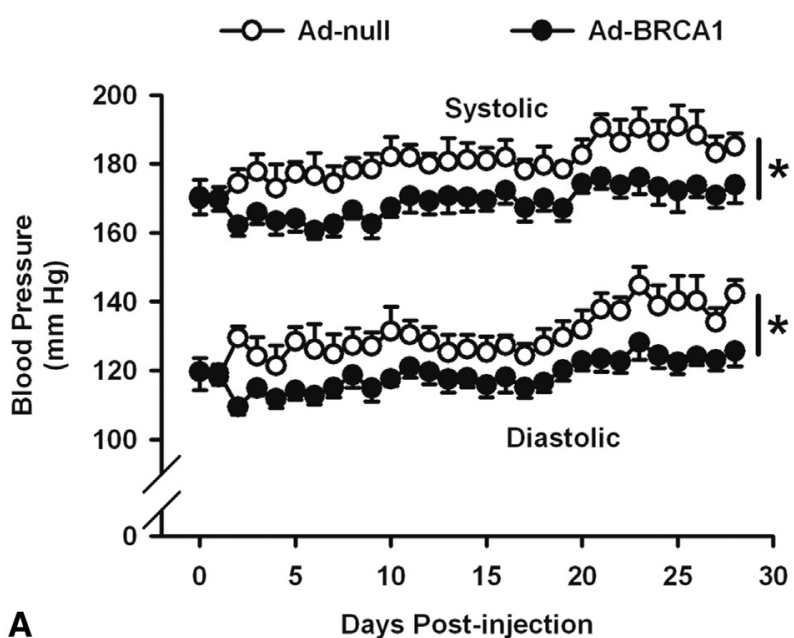

A

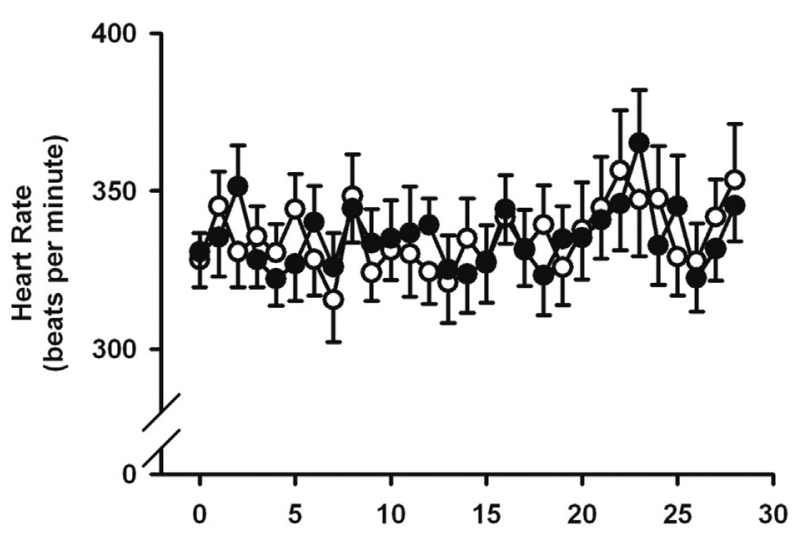

B

Days Post-injection

FIGURE 5. BRCA1 overexpression lowers systolic and diastolic blood pressure in SHR rats. A, Blood pressure and (B) heart rates were continuously recorded via radiotelemetry in SHR rats treated with either Ad-null or Ad-BRCA1 $\left(5 \times 10^{10} \mathrm{IU} / \mathrm{rat}\right.$, intravenously $) . \mathrm{n}=9$ per group. $* P<.05$ versus Ad-null. Ad-null, Control adenovirus; Ad-BRCA1, BRCA1 adenovirus.

systemic BRCA1 gene therapy would lower aortic ROS production and in turn attenuate blood pressure in SHR rats. The adequacy of adenovirus delivery was confirmed by demonstrating that BRCA1 expression was higher in livers harvested from SHR rats 1 week after Ad-BRCA1 or Ad-null administration (Figure E3, A) and that 4 weeks after adenovirus administration, aortic BRCA1 transcript levels were markedly higher in SHR rats treated with Ad-BRCA1 compared with Ad-null (Figure E3, B).

Ad-BRCA1 therapy resulted in an early and sustained reduction in both systolic and diastolic blood pressure without any effect on heart rate (Figure 5). The average systolic and diastolic blood pressure was appreciably lower in animals injected with Ad-BRCA1 compared with Adnull (systolic: Ad-null, $180.6 \pm 4.8 \mathrm{~mm} \mathrm{Hg}$; Ad-BRCA1, $169.2 \pm 3.8 \mathrm{~mm} \mathrm{Hg}, P<.05$; diastolic: Ad-null, $130.5 \pm$ $4.9 \mathrm{~mm} \mathrm{Hg}$; Ad-BRCA1, $119.3 \pm 3.3 \mathrm{~mm} \mathrm{Hg}, P<.05)$.
ROS production, as evaluated by lucigenin-enhanced chemiluminescence, was appreciably lower in aortas from SHR rats treated with Ad-BRCA1 compared with Ad-null (Figure 6, A). Aorta homogenates from SHR rats treated with Ad-BRCA1 (vs Ad-null) demonstrated lower NADPH oxidase activity (Figure $6, B$ ) and revealed less Nox 1 and p47phox protein (Figure 6, C). Levels of $\gamma \mathrm{H} 2 \mathrm{~A} . \mathrm{X}$, an end point of ROS-associated double-strand breaks (DSBs), was higher in the SMC nuclei of aortas from SHR rats than in those from WKY rats (Figure 7). The enhanced $\gamma \mathrm{H} 2 \mathrm{~A}$.X levels were notably absent in aortic samples from SHR rats that had previously received BRCA1 gene therapy (Figure 7). Furthermore, RAD51 foci formation, an indicator of DNA repair, was significantly lower in SMCs from SHR rat aortas than in SMCs from WKY rat and Ad-BRCA1-treated SHR rat aortas (Figure 8).

\section{DISCUSSION}

The present study identifies a previously unrecognized role of BRCA1 as a potential target to shield VSMCs from oxidative stress in vitro and in vivo. This study demonstrates that (1) BRCA1 is basally expressed in rat, murine, and human VSMCs; (2) prooxidants such as $\mathrm{H}_{2} \mathrm{O}_{2}$ reduce BRCA1 expression in VSMCs; (3) in vitro gain-of-function strategies with Ad-BRCA1 overexpression reduce $\mathrm{H}_{2} \mathrm{O}_{2}$-induced $\mathrm{ROS}$ production via a downregulation of Nox1 and its regulatory subunit p47phox; (4) VSMCs isolated from hypertensive SHR rats exhibit an increase in NADPH oxidase activity and ROS generation, which is attenuated by in vivo gene therapy with Ad-BRCA1; and (5) in vivo gene therapy with Ad-BRCA1 causes a reduction in blood pressure in SHR rats. In addition to extending our current understanding of BRCA1-mediated cellular protection, ${ }^{10-14}$ these data provide a translational link between ROS, DNA repair genes, and aberrant VSMC regulation.

ROS have been implicated as a central mechanism through which VSMCs undergo pathologic cellular activation resulting in aberrant phenotypic modulation, differentiation, migration, proliferation, extracellular matrix production, and cytoskeletal rearrangement. These pathologic changes in VSMCs have been causally linked to the development of various vascular diseases, including atherosclerosis, restenosis, neointimal hyperplasia, and aneurysm formation. ${ }^{1-7}$

NADPH oxidases are an important source of vascular ROS. ${ }^{1,2}$ The NADPH oxidase family comprises 7 Nox isoforms (Nox1-5, Duox 1, and Duox2), which function primarily as ROS-generating enzymes, leading to the production of superoxide and other free radicals. The Nox family represents transmembrane-associated proteins that transfer electrons across membranes such that the final electron acceptor is $\mathrm{O}_{2}$ and superoxide is generated. 

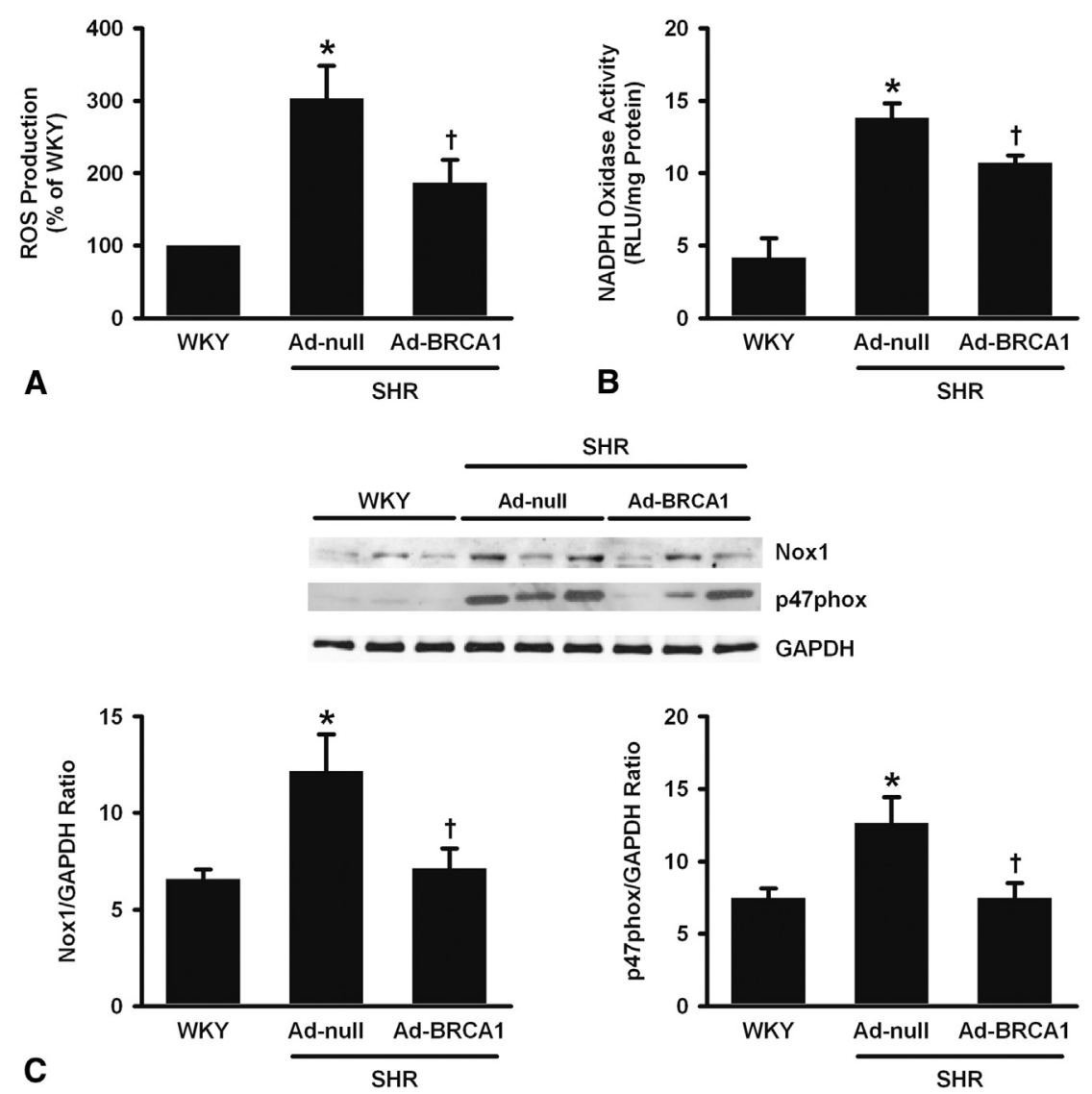

FIGURE 6. BRCA1 overexpression reduces aortic oxidative stress in SHR rats via the NADPH oxidase system. A, ROS production ( $\mathrm{n}=10$ per group) and (B) NADPH oxidase activity ( $\mathrm{n}=6$ per group) in aorta homogenates from WKY and adenovirus-treated SHR rats were assessed by lucigenin-enhanced chemiluminescence. C, Representative Western blots and semiquantitative data for the NADPH oxidase subunits Nox1 and p47phox in WKY and adenovirustreated SHR rat aortas. GAPDH acted as the housekeeping protein. $\mathrm{n}=6$ per group. ${ }^{*} P<.05$ versus WKY group; $\dagger P<.05$ versus Ad-null SHR group. $R O S$, Reactive oxygen species; WKY, Wistar-Kyoto rat; Ad-null, control adenovirus; Ad-BRCA1, BRCA1 adenovirus; SHR, spontaneously hypertensive rat; $R L U$, relative light units; $G A P D H$, glyceraldehyde-3-phosphate dehydrogenase; $N A D P H$, nicotinamide adenine dinucleotide phosphate.

For every 1 NADPH reduced, 2 superoxide molecules are created. All Nox isoforms have a core catalytic unit and several core regulatory units, which include 2 organizer subunits (p47phox and Noxo1), 2 activator subunits (p67phox and Noxa1), and p22phox and p40phox. ${ }^{1,2}$

Nox1 is found abundantly in SMCs and in colonic epithelium, endothelium, fibroblasts, cardiomyocytes, and microglia. ${ }^{1}$ It requires the regulatory unit $\mathrm{p} 47 \mathrm{phox}$ for its activity, in addition to other units including p22phox and p67phox. Studies demonstrate that Nox1 may represent a common mechanism linking various risk factors with VSMC dysfunction. Key mechanical and biochemical mediators of VSMC activation, such as angiotensin II, aldosterone, platelet-derived growth factor, epidermal growth factor, and altered mechanical/shear stress, have been shown to upregulate Nox 1 and induce oxidative stress in VSMCs. ${ }^{1,15,16}$ Additional studies have implicated Nox1 as a critical regulator of VSMC migration, proliferation, and phenotypic modulation, and Nox1 expression is increased in various cardiovascular disease states, including hypertension, atherosclerosis, diabetes, and hypercholesterolemia. ${ }^{1,17-19}$ Angiotensin II, a key mediator of vascular diseases, has been demonstrated to transduce its pressor and proinflammatory effects, in part through Nox $1 .{ }^{20}$ Several key transcription factors are upregulated by Nox-derived ROS including G protein-coupled receptor, Notch, Wnt- $\beta$-catenin, MAPK, Janus kinase/ signal transducers and activators of transcription, nuclear factor kappa B, phosphoinositide-3-kinase/protein kinase B, hypoxia-inducible factor 1-alpha, activator protein-1, Rho kinase, and protein tyrosine phosphatases.

The BRCA1 gene is classified as a tumor suppressor and caretaker because of its proposed genome integrity maintenance functions. ${ }^{8,9}$ Germ-line mutations in BRCA1 predominantly predispose carriers to breast and ovarian cancer in addition to other cancer syndromes. ${ }^{21}$ BRCA1 is important for various cellular functions and exerts its effects on key cellular processes involved in DNA nucleotide excision and DSB repair, transcriptional regulation, ubiquitination, and cell cycle control. ${ }^{8,9}$ Mice harboring 


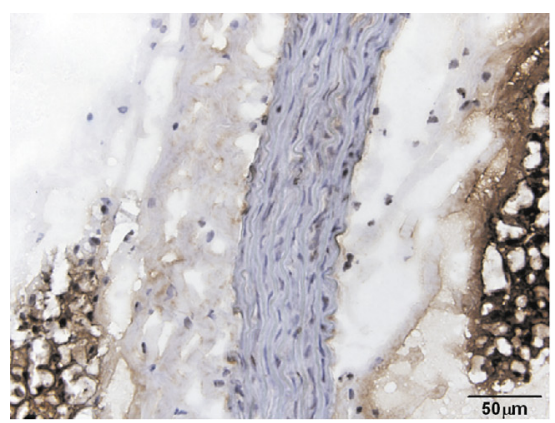

WKY

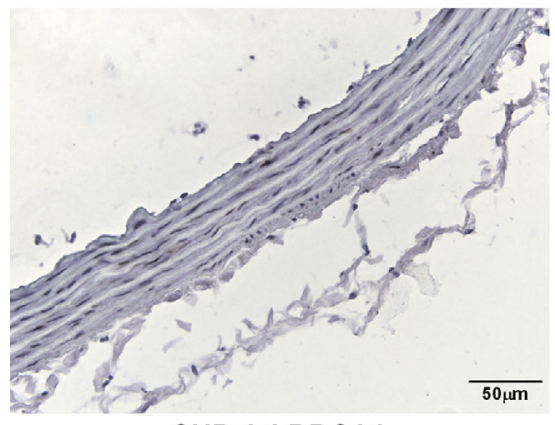

SHR Ad-BRCA1
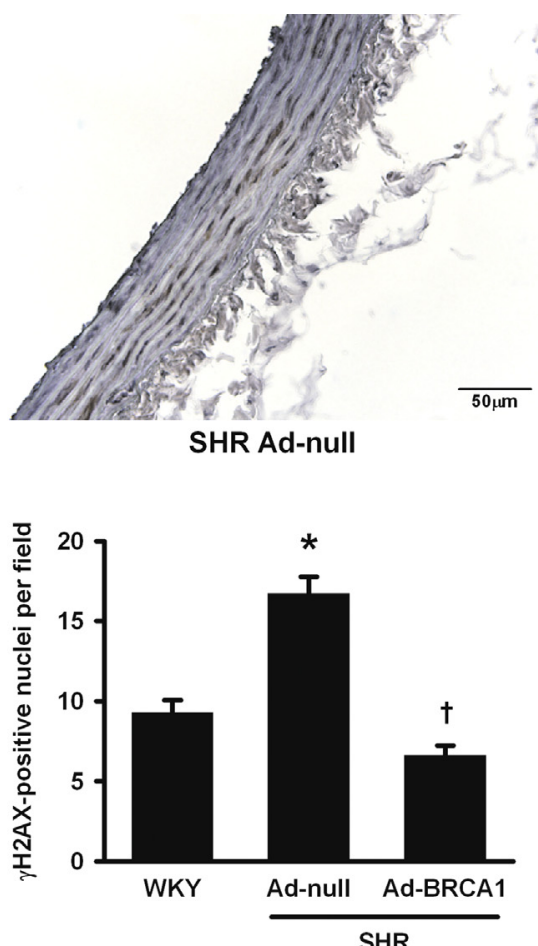

FIGURE 7. Aortas from Ad-BRCA1-treated SHR rats exhibit less H2A.X phosphorylation than those from Ad-null-treated SHR rats. $\gamma H 2 A . X$ levels were assessed via immunohistochemistry. Images are representative of $\mathrm{n}=5$ per group. $* P<.05$ versus WKY group; $\dagger P<.05$ versus Ad-null SHR group. $W K Y$, Wistar-Kyoto rat; SHR, spontaneously hypertensive rat; Ad-null, control adenovirus; Ad-BRCA1, BRCA1 adenovirus.

complete ablation of BRCA1 die embryonically and display neuroepithelial, proliferative, and morphogenetic abnormalities. The BRCA1 gene is composed of 24 exons. ${ }^{22,23}$ Exon 11 is the largest exon and covers $\sim 60 \%$ of the entire BRCA1 gene. ${ }^{21}$ The exon 11-encoded domain interacts and colocalizes with RAD51 at the sites of DNA damage to activate RAD51-mediated repair of DSBs. ${ }^{21,24}$ Mice with systemic deletion of exon 11 in the BRCA1 gene show recessive embryonic lethality as a result of impaired DNA damage-induced activation of BRCA1, a compromised RAD51-dependent repair pathway, and increased susceptibility to oxidative stress and apoptosis. ${ }^{25}$

Unopposed activation of ROS eventually leads to DNA damage, which is the common mechanism through which cellular apoptosis and/or necrosis develop. Because BRCA1 is widely known for its effects in promoting DNA damage repair, particularly in cancer cells, we reasoned that it may also be an important cellular defense mechanism against oxidative stress in VSMCs. Our observations point toward an important and reciprocal relationship between BRCA1 and oxidative stress in VSMCs. Under basal conditions, increasing concentrations of $\mathrm{H}_{2} \mathrm{O}_{2}$, a prototypical inducer of oxidative stress, inhibited BRCA1 expression. Forced overexpression of BRCA1 in vitro upregulated NADPH oxidase Nox1 and its regulatory subunit p47phox, and inhibited $\mathrm{H}_{2} \mathrm{O}_{2}$-induced ROS production. To confirm the translational significance of these data, we used intravenous adenoviral delivery of BRCA1 to overexpress BRCA1 systemically in SHR rats, an established model of hypertension, wherein altered NADPH-related increases in ROS have been suggested to be causally related to the development of high blood pressure. In addition to a reduction in aortic VSMCs Nox 1 and p47phox expression and diminished ROS generation, systemic BRCA1 therapy not only prevented an increase in $\gamma \mathrm{H} 2 \mathrm{~A}$.X levels and increased RAD51 foci formation in SHR rat aortas but also attenuated systolic and diastolic blood pressure in these animals, with an effect that was noticed early, and was sustained over the 4-week observation period.

The limitations of this study must be highlighted. Increased ROS generation is only 1 mechanism of hypertension development in SHR rats, and other potential mechanisms such as sympathetic stimulation, alterations in renal regulatory pathways, or endothelial dysfunction were not evaluated. Because systemic administration was not SMC specific, an alternative antihypertensive mechanism cannot be ruled out. Previous studies have demonstrated a beneficial effect of BRCA1 on endothelial function, ${ }^{11}$ and hence the combined effects on both endothelial and VSMC pathways may be operative. There was no change in heart rate, suggesting that the reduction in blood pressure was not secondary to a negative chronotropic effect. Although we assessed the translational significance of our 

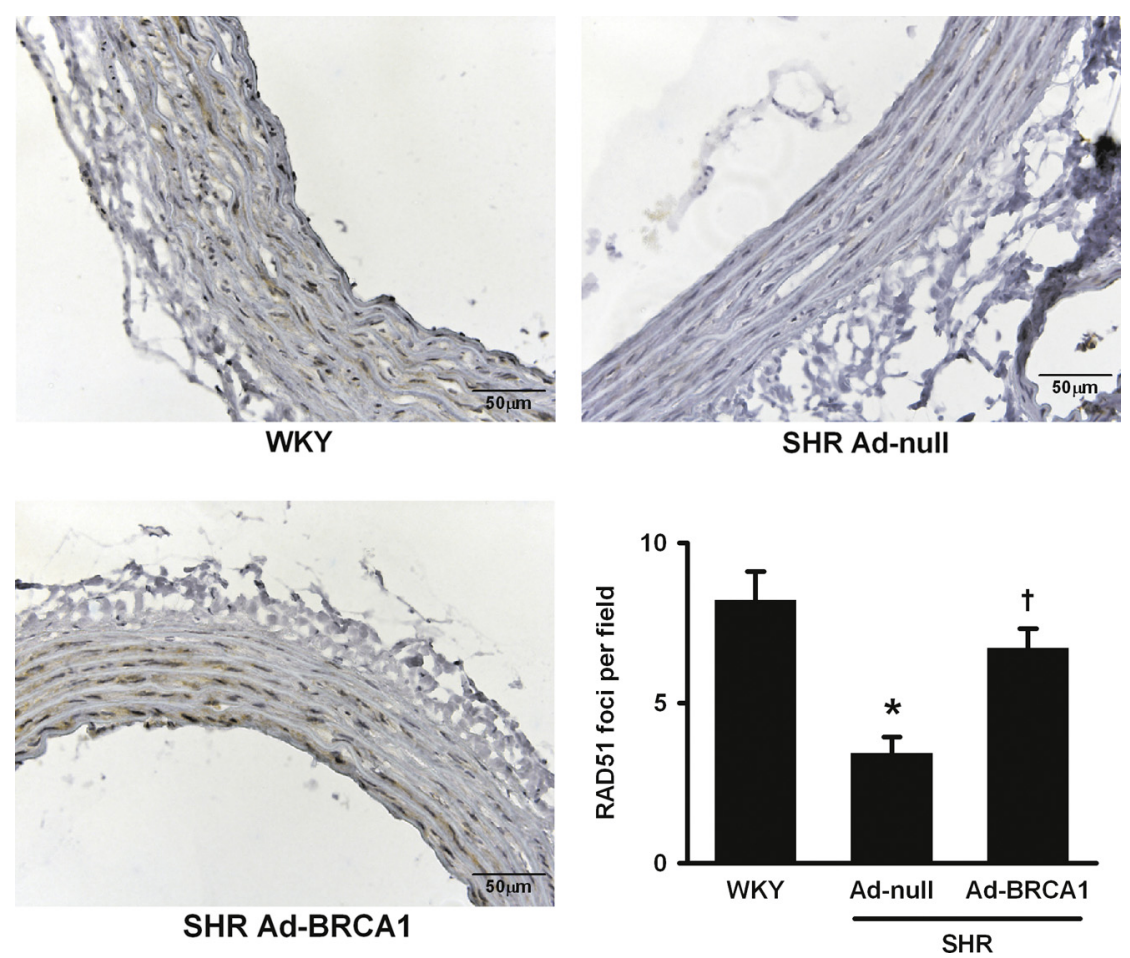

FIGURE 8. Aortas from Ad-BRCA1-treated SHR rats exhibit more RAD51 foci formation than those from Ad-null-treated SHR rats. RAD51 foci were quantified via immunohistochemistry. Images are representative of $\mathrm{n}=5$ per group. $* P<.05$ versus WKY group; $\dagger P<.05$ versus Ad-null SHR group. $W K Y$, Wistar-Kyoto rat; SHR, spontaneously hypertensive rat; Ad-null, control adenovirus; Ad-BRCA1, BRCA1 adenovirus.

in vitro findings in a model of experimental hypertension, we appreciate that VSMC may play a role in many diverse diseases including restenosis, neointimal injury, and aneurysm formation. Studies using BRCA1-based cell or gene therapy in such settings may be considered. ${ }^{26-29}$

The authors thank Praphulla C. Shukla for assistance with the tail vein injections.

\section{References}

1. Montezano AC, Touyz RM. Reactive oxygen species, vascular Noxs, and hypertension: focus on translational and clinical research. Antioxid Redox Signal. June 6, 2013 [Epub ahead of print].

2. Zhou Y, Yan H, Guo M, Zhu J, Xiao Q, Zhang L. Reactive oxygen species in vascular formation and development. Oxid Med Cell Longev. 2013;2013: 374963.

3. Sehgel NL, Zhu Y, Sun Z, Trzeciakowski JP, Hong Z, Hunter WC, et al. Increased vascular smooth muscle cell stiffness; a novel mechanism for aortic stiffness in hypertension. Am J Physiol Heart Circ Physiol. 2013;305:H1281-7.

4. Lacolley P, Regnault V, Nicoletti A, Li Z, Michel JB. The vascular smooth muscle cell in arterial pathology: a cell that can take on multiple roles. Cardiovasc Res. 2012;95:194-204.

5. Lovren F, Pan Y, Quan A, Singh KK, Shukla PC, Gupta N, et al. MicroRNA-145 targeted therapy reduces atherosclerosis. Circulation. 2012;126:S81-90.

6. Denes L, Entz L, Jancsik V. Restenosis and therapy. Int J Vasc Med. 2012;2012: 406236.

7. Della Corte A, Quarto C, Bancone C, Castaldo C, Di Meglio F, Nurzynska D, et al. Spatiotemporal patterns of smooth muscle cell changes in ascending aortic dilatation with bicuspid and tricuspid aortic valve stenosis: focus on cell-matrix signaling. J Thorac Cardiovasc Surg. 2008;135:8-18. e1-2.

8. Greenberg RA. Recognition of DNA double strand breaks by the BRCA1 tumor suppressor network. Chromosoma. 2008;117:305-17.
9. Sobhian B, Shao G, Lilli DR, Culhane AC, Moreau LA, Xia B, et al. RAP80 targets BRCA1 to specific ubiquitin structures at DNA damage sites. Science. 2007;316:1198-202.

10. Shukla PC, Singh KK, Quan A, Al-Omran M, Teoh H, Lovren F, et al. BRCA1 is an essential regulator of heart function and survival following myocardial infarction. Nat Commun. 2011;2:593.

11. Singh KK, Shukla PC, Quan A, Al-Omran M, Lovren F, Pan Y, et al. BRCA1 is a novel target to improve endothelial dysfunction and retard atherosclerosis. J Thorac Cardiovasc Surg. 2013;146:949-60.e4.

12. Singh KK, Shukla PC, Quan A, Desjardins JF, Lovren F, Pan Y, et al. BRCA2 protein deficiency exaggerates doxorubicin-induced cardiomyocyte apoptosis and cardiac failure. J Biol Chem. 2012;287:6604-14.

13. Singh KK, Shukla PC, Yanagawa B, Quan A, Lovren F, Pan Y, et al. Regulating cardiac energy metabolism and bioenergetics by targeting the DNA damage repair protein BRCA1. J Thorac Cardiovasc Surg. 2013;146: 702-9.

14. Teoh H, Quan A, Creighton AK, Annie Bang KW, Singh KK, Shukla PC, et al. BRCA1 gene therapy reduces systemic inflammatory response and multiple organ failure and improves survival in experimental sepsis. Gene Ther. 2013; 20:51-61.

15. Manea A, Tanase LI, Raicu M, Simionescu M. Transcriptional regulation of NADPH oxidase isoforms, Nox1 and Nox4, by nuclear factor-kappaB in human aortic smooth muscle cells. Biochem Biophys Res Commun. 2010;396:901-7.

16. Pescatore LA, Bonatto D, Forti FL, Sadok A, Kovacic H, Laurindo FR. Protein disulfide isomerase is required for platelet-derived growth factor-induced vascular smooth muscle cell migration, Nox1 NADPH oxidase expression, and RhoGTPase activation. J Biol Chem. 2012;287:29290-300.

17. Dikalova AE, Gongora MC, Harrison DG, Lambeth JD, Dikalov S, Griendling KK. Upregulation of Nox1 in vascular smooth muscle leads to impaired endothelium-dependent relaxation via eNOS uncoupling. Am J Physiol Heart Circ Physiol. 2010;299:H673-9.

18. Sheehan AL, Carrell S, Johnson B, Stanic B, Banfi B, Miller FJ Jr. Role for Nox1 NADPH oxidase in atherosclerosis. Atherosclerosis. 2011;216:321-6.

19. Valente AJ, Yoshida T, Murthy SN, Sakamuri SS, Katsuyama M, Clark RA, et al. Angiotensin II enhances AT1-Nox1 binding and stimulates arterial smooth 
muscle cell migration and proliferation through AT1, Nox1, and interleukin-18. Am J Physiol Heart Circ Physiol. 2012;303:H282-96.

20. Matsuno K, Yamada H, Iwata K, Jin D, Katsuyama M, Matsuki M, et al. Nox1 is involved in angiotensin II-mediated hypertension: a study in Nox1-deficient mice. Circulation. 2005;112:2677-85.

21. Xu X, Wagner KU, Larson D, Weaver Z, Li C, Ried T, et al. Conditional mutation of Brcal in mammary epithelial cells results in blunted ductal morphogenesis and tumour formation. Nat Genet. 1999;22:37-43.

22. Casey G. The BRCA1 and BRCA2 breast cancer genes. Curr Opin Oncol. 1997; 9:88-93.

23. Miki Y, Swensen J, Shattuck-Eidens D, Futreal PA, Harshman K, Tavtigian S, et al. A strong candidate for the breast and ovarian cancer susceptibility gene BRCA1. Science. 1994;266:66-71.

24. Scully R, Livingston DM. In search of the tumour-suppressor functions of BRCA1 and BRCA2. Nature. 2000;408:429-32.
25. Hakem R, de la Pompa JL, Mak TW. Developmental studies of Brca1 and Brca2 knock-out mice. J Mammary Gland Biol Neoplasia. 1998;3:431-45.

26. Zbuk K, Xie C, Young R, Heydarpour M, Pare G, Davis AD, et al BRCA2 variants and cardiovascular disease in a multi-ethnic study. BMC Med Genet. 2012;13:56.

27. Mai PL, Chatterjee N, Hartge P, Tucker M, Brody L, Struewing JP, et al Potential excess mortality in BRCA1/2 mutation carriers beyond breast, ovarian, prostate, and pancreatic cancers, and melanoma. PLoS One. 2009;4: e4812.

28. Bordeleau L, Lipscombe L, Lubinski J, Ghadirian P, Foulkes WD, Neuhausen S et al. Diabetes and breast cancer among women with BRCA1 and BRCA2 mutations. Cancer. 2011;117:1812-8.

29. Ozaki K, Sato H, Inoue K, Tsunoda T, Sakata Y, Mizuno H, et al. SNPs in BRAP associated with risk of myocardial infarction in Asian populations. Nat Genet. 2009;41:329-33. 


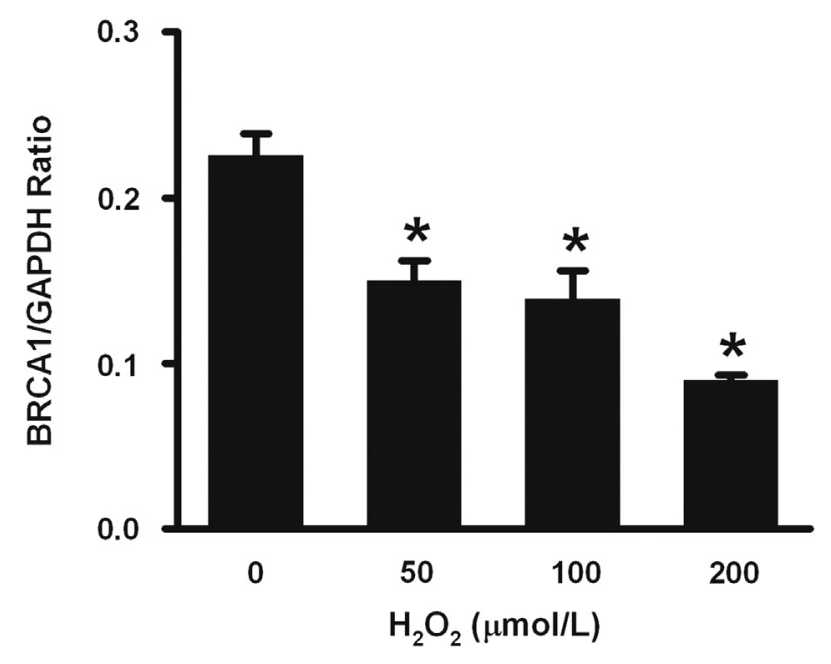

FIGURE E1. $\mathrm{H}_{2} \mathrm{O}_{2}$ reduces HASMC BRCA1 expression in a concentration-dependent manner. HASMCs were incubated with increasing concentrations of $\mathrm{H}_{2} \mathrm{O}_{2}$ for 24 hours before being processed for qPCR analysis. $\mathrm{n}=5$ per group. $* P<.05$ versus group not exposed to $\mathrm{H}_{2} \mathrm{O}_{2}$. BRCA1, Breast cancer 1, early onset gene; $G A P D H$, glyceraldehyde-3-phosphate dehydrogenase.

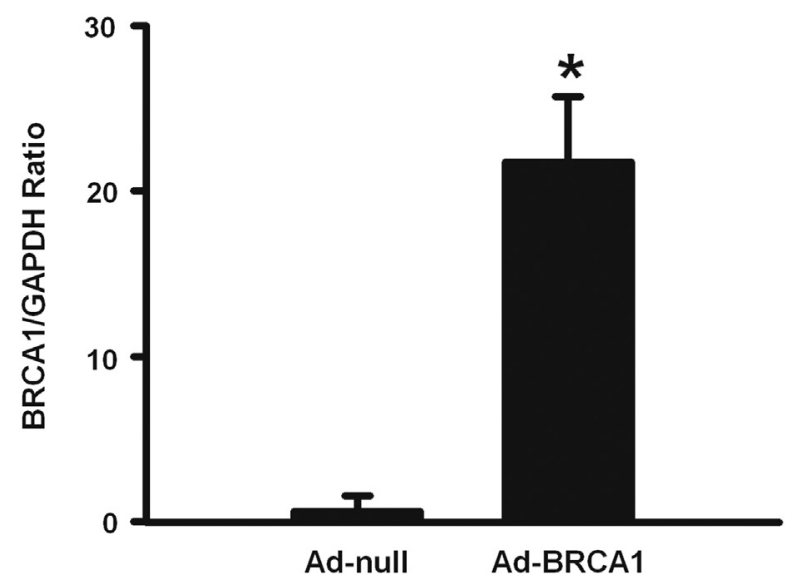

FIGURE E2. BRCA1 is overexpressed in SHR RASMCs transfected with Ad-BRCA1. BRCA1 transcripts in adenovirus-transfected (10 MOI) SHR RASMCs were evaluated by qPCR. $\mathrm{n}=5$ per group. ${ }^{*} P<.05$ versus Ad-null group. BRCA1, Breast cancer 1, early onset gene; GAPDH, glyceraldehyde-3-phosphate dehydrogenase; Ad-null, control adenovirus; Ad-BRCA1, BRCA1 adenovirus.
LIVER

(1 week after adenovirus administration)

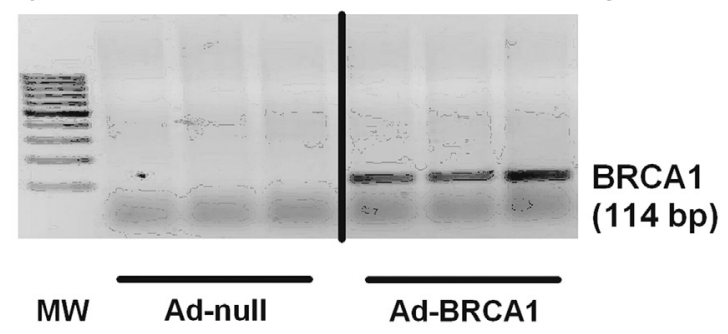

AORTA

(4 weeks after adenovirus administration)

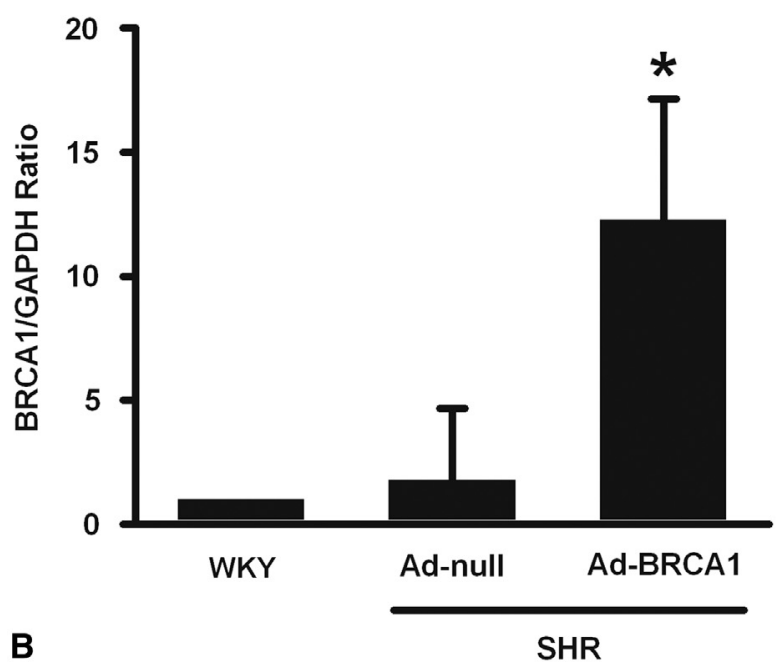

FIGURE E3. BRCA1 is overexpressed in the livers and aortas of SHR rats after treatment with Ad-BRCA1. SHR rats were administered either Ad-null or Ad-BRCA1 $\left(5 \times 10^{10} \mathrm{IU} / \mathrm{rat}\right.$, intravenously). A, Representative agarose gel images of BRCA1 PCR products of livers from SHR rats 1 week after adenovirus administration. $\mathrm{n}=7$ per group. Solid line demarcates nonadjacent lanes from the same gel. B, qPCR results for BRCA1 transcripts in aortas from SHR rats 4 weeks after adenovirus administration. $\mathrm{n}=7$ per group. ${ }^{*} P<.05$ versus Ad-null. $M W$, Molecular weight marker; BRCA1, Breast cancer 1, early onset gene; Ad-null, control adenovirus; Ad-BRCA1, BRCA1 adenovirus; GAPDH, glyceraldehyde-3phosphate dehydrogenase; $W K Y$, Wistar-Kyoto rat; $S H R$, spontaneously hypertensive rat. 